\title{
DEGRADATION OF MULTIBUNCH LUMINOSITY IN A LINEAR COLLIDER DUE TO CUMULATIVE BEAM BREAKUP*
}

\author{
C. L. Bohn, M. Syphers, FNAL, Batavia, IL 60510, USA \\ D. Schulte, CERN, Geneva, Switzerland
}

\begin{abstract}
Beam-excited transverse wakes in accelerating radiofrequency structures will influence the transverse offsets of each bunch in a multibunch train, causing the projected emittance of the bunch train to grow. An analytic theory of this phenomenon that includes the mitigating influence of a correlated energy spread across the bunch train was recently devised and applied to electron-positron linear colliders. We use the results of this theory to estimate analytically the associated degradation of multibunch luminosity in terms of toplevel parameters for the two beams, the two accelerators, and the final-focus system. Then we compare the estimates with results from GUINEA-PIG, a code that includes the detailed physics of beam-beam interactions.
\end{abstract}

\section{INTRODUCTION}

We recently developed an analytic theory of cumulative multibunch beam breakup (MBBU) that includes a linear variation of transverse focusing across the bunch train [1]. The focusing variation saturates the exponential growth of the beam breakup and establishes an algebraic decay of the transverse bunch displacement versus bunch number. However, particularly in the case of a beam that is misaligned at injection, the bunch train at linac exit may exhibit a relic head-to-tail betatron oscillation caused by the focusing variation [2]. Consequently, the bunch train itself may assume a complicated form.

Because a linear collider brings bunch trains from two distinct linacs into collision, the final-focus system must ensure the bunch-to-bunch overlap at the interaction point is sufficient to achieve the desired multibunch luminosity. The nominally $2.8 \mathrm{~ns}$ bunch spacing envisioned for the Next Linear Collider (NLC) is short, making it difficult to do interbunch feedback to bring bunch pairs into collision. Consequently the effect of the focusing variation itself on the multibunch luminosity is important. As shown here, by considering only the geometric overlap of the bunch pairs, one can devise analytic estimates of multibunch luminosity that combine the parameters of the two beams, the two linacs, and the final focus. These estimates do not include complications of beam-beam interactions, and we explore the impact of this physics via simulations. Implicit in our treatment is the notion that single-bunch BBU has already been cured via, e.g., BNS damping.

*Work supported by Universities Research Association, Inc., under contract DE-AC02-76CH00300 with the U.S. Department of Energy.

\section{ANALYTIC ESTIMATE}

An analytic expression for the MBBU envelope containing all of the bunches comprising a bunch train is given in Refs. [1,2]. The expression includes a linear variation of transverse focusing (a form of BNS damping) along the bunch train. It is based on a continuum form of the equation of motion of point bunches in which the discrete transverse kicks imparted by the radiofrequency (rf) structures are smoothed along the linac. Refs. [1,2] also validate the analytic solution against numerical solutions of the equation of motion.

Modeling each bunch as being one-dimensional, i.e., flat, with a gaussian transverse density distribution, and accounting for the centroids of colliding bunch pairs to be offset at the interaction point (IP), we convolve the density distributions of each colliding pair, sum over all $M$ pairs, and obtain the "luminosity degradation"

$$
\frac{L}{L_{0}}=\frac{1}{M} \sum_{m=0}^{M-1} \exp \left[-\frac{\left(Y_{m}-Y_{-m}\right)^{2}}{4 \sigma^{2}}\right] \leq 1,
$$

in which $Y_{m}$ and $Y_{-m}$ denote the centroid positions of the positron and electron bunches, respectively, comprising the $m^{\text {th }}$ pair, and $\sigma$ denotes the rms transverse bunch halfwidth. Eq. (1) is the basis for deriving the luminosity degradation from a numerical solution of the equation of transverse motion.

To obtain an analytic estimate in terms of the aforementioned envelope which we shall denote as $\left|y_{m}\right|$ for the $m^{\text {th }}$ bunch in a train, we resort to a gaussian-statistical analysis. Taking the bunch centroids to be normally distributed about the displacement they would have were BBU zero, and taking the centroids of the lead bunches to be offset from one another by the distance $\chi$, we obtain

$$
\begin{aligned}
& \frac{L}{L_{0}}=\frac{\sigma \sqrt{2}}{M} \sum_{m=0}^{M-1} \frac{1}{S_{T}(m)} \exp \left(-\frac{\chi^{2}}{2 S_{T}^{2}(m)}\right) ; \\
& S_{T}(m)=\sqrt{2 \sigma^{2}+\Sigma_{p}^{2}(m)+\Sigma_{e}^{2}(m)} ;
\end{aligned}
$$

in which $\Sigma_{p}(m)$ and $\Sigma_{e}(m)$ refer to the analytically determined envelopes $\left|y_{m}\right|$ of the positron and electron beams, respectively. They will generally differ in keeping with the parameters of the two linacs and beams, and Eq. (2) implicitly includes these distinctions. For the special case of zero MBBU, for which $\Sigma_{p}(m)=\Sigma_{e}(m)=0$, Eq. (2) reduces to Eq. (1), as it should.

Two illustrative examples are provided in Figs. 1 and 2. Table 1 lists the parameters used to generate these figures. 
They are consistent with NLC specifications; however, the wake amplitude and quality factor are pessimistically chosen to replicate the consequences of a hypothetically off-nominal wake. Were, e.g., the NLC rf-structure design specifications strictly achieved, then MBBU would be insignificant. Figs. 1 and 2 show the bunch trains, presumed to be missteered through the final-focus system such that the lead bunches are offset at the IP by an amount $\chi$. The bunch-train offsets (and angles) are calculated numerically from the equation of motion given $0 \%$ and $3 \%$ energy spread, respectively. The positron beam was derived for these specific examples by simply multiplying the bunch displacements of the electron beam by -1 . The luminosity degradation calculated numerically from Eq. (1) and analytically from Eq. (2) is also shown.

These two examples suggest the analytic calculation is reasonably accurate. We have checked many cases, including randomized parameters, different bunch-train geometries, and nonidentical colliding beams. As long as the MBBU is not too large compared to the rms bunch size, the analytic solution stays reasonably accurate. Otherwise, it cannot replicate the fine structure in the density profile at large displacements.

Table 1: Hypothetical Linear Collider Parameters

\begin{tabular}{|l|l|}
\hline Parameter & Value \\
\hline initial electron-beam offset & $2.2 \mu \mathrm{m}$ \\
\hline initial positron-beam offset & $-2.2 \mu \mathrm{m}$ \\
\hline injected angle of beams & $0 \mathrm{deg}$ \\
\hline injected beam kinetic energy & $8 \mathrm{GeV}$ \\
\hline output beam kinetic energy & $250 \mathrm{GeV}$ \\
\hline length of each linac & $4.5 \mathrm{~km}$ \\
\hline bunch charge & $1.3 \mathrm{nC}$ \\
\hline $\begin{array}{l}\text { bunch train ("macropulse") } \\
\text { format }\end{array}$ & $90 \mathrm{bunches}$ separated \\
\hline by $2.8 \mathrm{nsec}$ \\
\hline number of betatron periods & 100 \\
\hline long-range wake amplitude & $1 \cdot 10^{15} \mathrm{~V} / \mathrm{C} / \mathrm{m}^{2}$ \\
\hline effective deflecting-wake $Q$ & $\infty$ \\
\hline deflecting-mode frequency & $14.95 \mathrm{GHz}$ \\
\hline beta function at IP & $0.1 \mathrm{~mm}$ \\
\hline final-focus demagnification & 349 \\
\hline rms bunch half-width at IP & $2.7 \mathrm{~nm}$ \\
\hline rms bunch length at IP & $110 \mu \mathrm{m}$ \\
\hline
\end{tabular}

Figs. 1 and 2 suggest the minimum luminosity loss for both $0 \%$ and $3 \%$ energy spread is about $40 \%$, which is not catastrophic despite the pessimistic parameters of the deflecting wake. Given the large energy spread of $3 \%$ (Fig. 2), the maximum luminosity is about the same as with no energy spread (Fig. 1), a counterintuitive finding. It is a signature of the "flip-orientation" of the bunch trains, i.e., one train having an injection offset opposite to that of the other train. However, "like-oriented" bunch trains can be constructed by, e.g., subtracting the betatron curvature from the bunch offsets of one train, multiplying
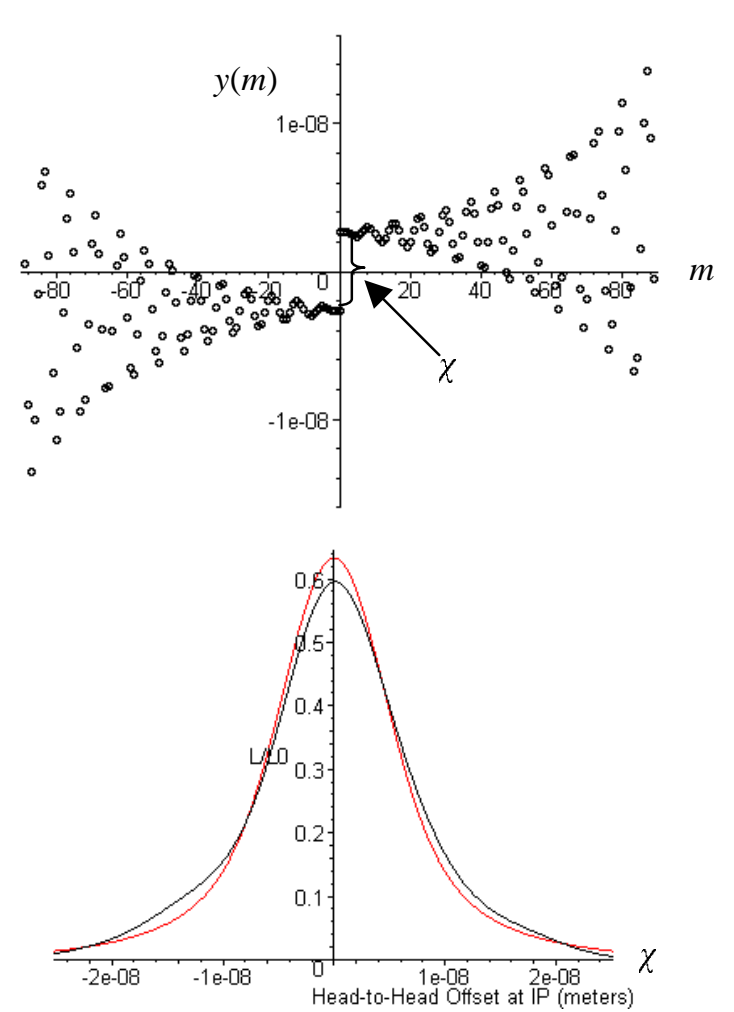

Figure 1. Zero energy spread. Top: Bunch trains, with lead bunches at IP. Bottom: Luminosity degradation vs. $\chi$ calculated analytically (red) and numerically (black).

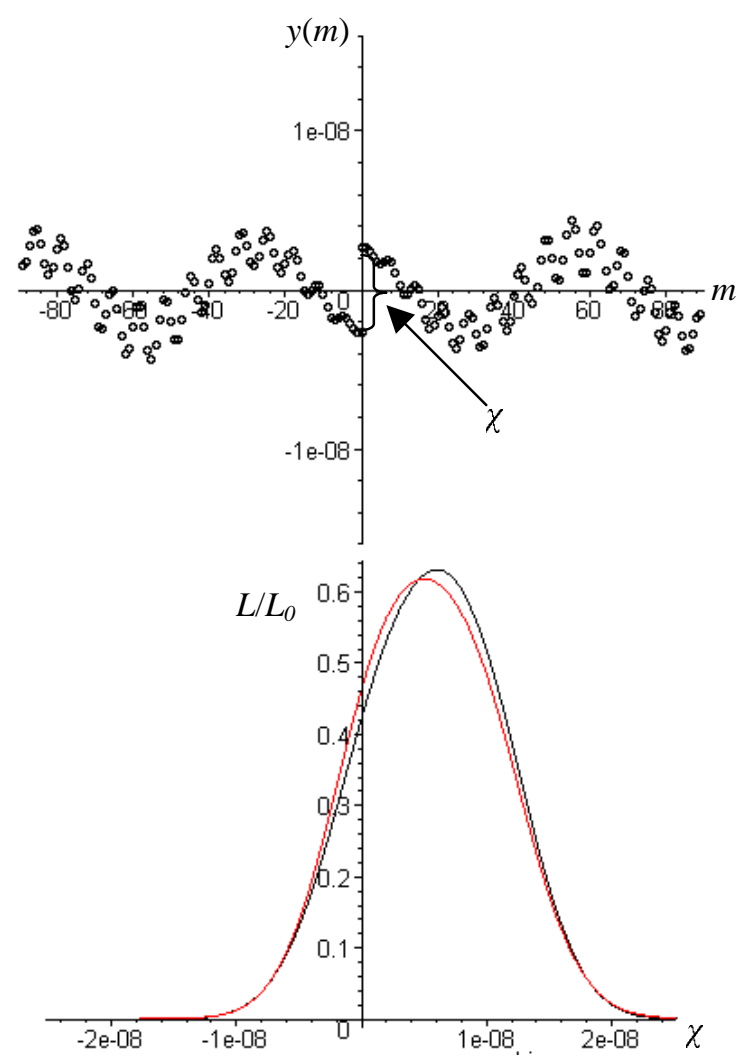

Figure 2. 3\% energy spread. Top: Bunch trains, with lead bunches at IP. Bottom: Luminosity degradation vs. $\chi$ calculated analytically (red) and numerically (black). 
the resulting offset by -1 , then adding back the betatron curvature to obtain the second bunch train. The minimum luminosity loss for these like-oriented trains is only about $10 \%$ for the case of $3 \%$ energy spread, which is one way of confirming the intuitive notion that damping the MBBU should normally improve the multibunch luminosity.

\section{BEAM-BEAM EFFECTS}

The preceding results were derived with the motivation of estimating the gross influence of relatively large MBBU on the luminosity. Only the geometric overlap of the transverse density distributions of the colliding bunches was considered; beam-beam effects were ignored. We now summarize results of more realistic simulations with the code GUINEA-PIG [3] that include these effects. The simulations are based on the parameters of Table 1.

Taking the vertical emittance at the interaction point to be $\varepsilon_{y}=35 \mathrm{~nm}$, we simulated collisions with various possible combinations of angle and position error at the IP. Figure 3 shows the luminosity degradation for two colliding bunches as a function of position error $\Delta y$ for collision without an angle error, and as a function of the angle error $\Delta y^{\prime}$ for collision without a position error. The simulations were performed first for rigid beams, and then taking the beam-beam forces into account. In both cases the hour-glass effect was included. The result for rigid beams is not much different from Eq. (1).

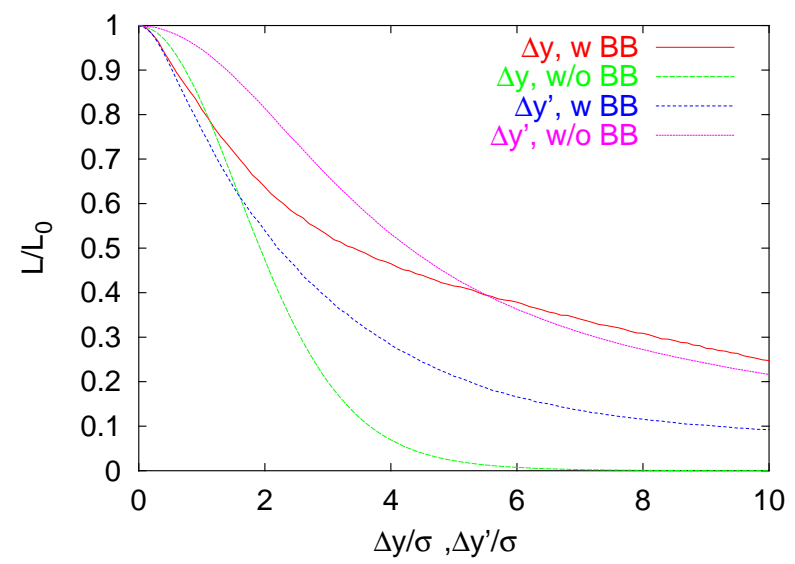

Figure 3. Luminosity degradation for two colliding bunches vs. offset $\Delta y$ and angle $\Delta y^{\prime}$, with and without beam-beam (BB) effects.

Beam-beam effects significantly enhance the sensitivity to small position errors but improve the luminosity given large position errors. The sensitivity to angle errors strongly increases over the full range displayed. Figure 3 suggests the totality of beam-beam effects would affect modestly, not pronouncedly, the analytic results of $\S 2$.

We now turn to a more realistic MBBU analysis, one involving simulations of a "perfect" machine wherein the injected beam offset is due to unavoidable jitter rather than systematic misalignment errors. The luminosity loss of the full train is determined by averaging over 100 different linacs having the parameters of Table 1 , but in which the beam offsets at injection are selected per a gaussian distribution having $\sigma=2.2 s \mu \mathrm{m}$, with $s$ being a free parameter. The bunch-centroid offsets of Figs. 1 and 2 then scale linearly with the chosen injection offsets. Any applied energy spread is considered to be perfectly removed near the end of the linac, prior to final focus. The luminosity loss is plotted versus $s \leq 0.3$ in Fig. 4, for which MBBU is thus relatively modest. The beam-beam interaction increases the luminosity loss over that of rigid beams, whether the beam is accelerated with an energy spread or not. For $s=0.3$, the luminosity loss is $\sim 13 \%$ and $\sim 7 \%$ with energy spreads of $0 \%$ and $3 \%$, respectively. Without beam-beam effects the results are $9 \%$ and $3 \%$, respectively. Were $s$ large enough to yield relatively large MBBU (as in the examples of \$2), the qualitative trend may change such that the beam-beam effects could then reduce the luminosity loss.

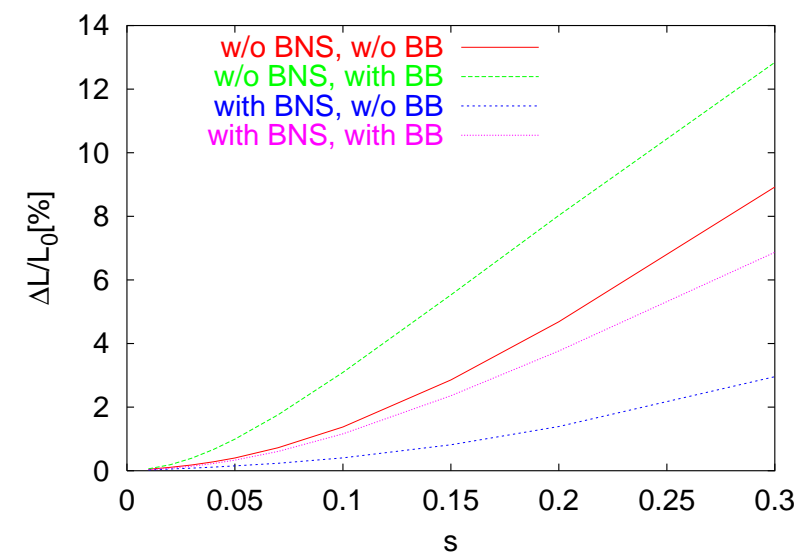

Figure 4. Luminosity loss $\Delta L / L_{0}$ vs. rms beam jitter $s$ at the linac entrances for energy spreads of $0 \%$ and $3 \%$.

\section{SUMMARY}

By considering only the geometric overlap of colliding bunches, we developed an analytic estimate of the luminosity degradation from multibunch beam breakup in a linear collider. The estimate includes top-level parameters of the two beams, the two linacs, and the finalfocus system. It does not include, however, effects from the beam-beam interaction during collision, nor does it include any single-bunch wakefield effects.

In realistic linear colliders, a multibunch energy spread should normally help, but beam-beam effects tend to increase the luminosity loss. However, no single-bunch BBU is included here, and it likewise will add to the luminosity loss. With small offsets, the luminosity with beam-beam effects is worse than without; at large offsets it is, however, better. Angle errors at collision add significantly to beam-beam-induced luminosity loss.

\section{REFERENCES}

[1] C. L. Bohn, K.-Y. Ng, PRL. 85, 984 (2000).

[2] C. L. Bohn, K.-Y. Ng, Proc. 2000 Linac Conf., SLAC-R-561, 31 (2000).

[3] D. Schulte, ICAP 1998, CERN-PS-99014 (1999). 